\title{
Unos sí y otros no: factores asociados a la recepción de apoyo familiar en las personas adultas mayores de tres entidades mexicanas
}

\section{Some yes and others no: factors associated with receiving family support in older persons in three Mexican entities}

\author{
ROSA MARÍA FLORES MARTÍNEZ \\ Facultad de Trabajo Social y Desarrollo Humano, Universidad Autónoma de Nuevo León rosamariaflores1@gmail.com \\ ORCID ID: 0000-0003-2432-124X
}

SAGRARIO GARAY VILLEGAS

Facultad de Trabajo Social y Desarrollo Humano, Universidad Autónoma de Nuevo León sgarayv@gmail.com ORCID ID: 0000-0002-9087-5526

\begin{abstract}
Resumen: México es en país que se encuentra en un pleno proceso de envejecimiento poblacional, lo cual representa un logro de las políticas sociales implementadas en las últimas décadas, al mismo tiempo, este fenómeno implica retos en materia de bienestar social en la vejez, especialmente en un contexto caracterizado por la incertidumbre. En ese sentido, el objetivo de este artículo es analizar los factores que intervienen en la recepción de apoyo familiar de las personas adultas mayores residentes en tres entidades del país: Nuevo León, Ciudad de México y Jalisco. La metodología es de corte cuantitativo, con un diseño transversal y exposfacto. La fuente de datos que se utiliza es la Encuesta sobre Condiciones de Vida de las Personas Adultas Mayores en México, 2016. Los principales resultados obtenidos revelan que las personas mayores que son mujeres, de una edad avanzada, que no trabajan, que sí tienen hijos y que viven en contextos rurales tienen una mayor probabilidad de recibir apoyo familiar. Por lo que se concluye que el apoyo familiar esta mediado por la convergencia de diversos factores, tanto individuales, como familiares y contextuales. De manera que habría que replantearse la distribución en la corresponsabilidad de apoyo en la vejez, pues ante un entorno de grandes cambios demográficos, sociales y económicos, la demanda de apoyo puede exceder la capacidad de las familias mexicanas.
\end{abstract}

Palabras clave: vejez, redes de apoyo, apoyo familiar, factores, bienestar social

\begin{abstract}
Mexico is in a country in process of population aging, which represents an achievement of the social policies implemented in recent decades, at the same time, this phenomenon implies challenges in terms of social welfare in old age, especially in a context characterized by uncertainty. The aim of this article is to analyze the factors involved in receiving family support from older persons living in three states of the country: Nuevo León, Mexico City and Jalisco. The methodology is quantitative, with a cross-sectional and expository design. The data source used is the Survey on Living Conditions of Older Persons in Mexico, 2016. The main results reveal that in the group of older persons those who are or
\end{abstract}


have: women, advanced age, do not working, have children and living in rural contexts are more likely to receive family support. It is therefore concluded that family support is mediated by the convergence of various individual, family and contextual factors. It would be necessary to rethink the distribution of co-responsibility for support in older ages, since in the face of major demographic, social and economic changes, the demand for support may exceed the capacity of Mexican families.

Key words: old age, support networks, family support, factors, social welfare

\section{INTRODUCCIÓN}

A nivel global el envejecimiento poblacional es un fenómeno que está marcado el presente siglo. De acuerdo con Help Age (2015) la población está envejeciendo, se estima que en el 2015 del total la población mundial 12.3\% eran personas de 60 años o más, asimismo se prevé que en el 2030 se incremente a $16.5 \%$ y en el 2050 se llegue a alcanzar la cifra de $21.5 \%$, no obstante, pese a este incremento las condiciones y experiencias de vida tienden a ser distintas según el país o la región en la que las personas mayores vivan.

Particularmente en el caso mexicano, según datos del Instituto Nacional de Estadística y Geografía, en el 2015, la población total fue de 119, 530, 753 habitantes, de los cuales $12,436,321$ eran personas de 60 años o más, lo que en términos relativos representa el $10.4 \%$ de la población total; así mismo, cabe señalar que del total de la población adulta mayor, las mujeres presentan una proporción más elevada (53.7\%).

Respecto a la esperanza de vida, las proyecciones del Consejo Nacional de Población (2018) muestran que para el año 2019 la esperanza de vida media estimada para la población mexicana es de 75 años, cabe señalar que en el caso de las mujeres el promedio es de 78.15 años, cifra superior a la esperanza de vida de los varones (71.98 años).

Aunque México es predominantemente joven, para el año 2030 la población de personas adultas mayores será de $14.9 \%$ y, en el 2050 aumentará aproximadamente a $24.7 \%$ (González, 2015). En general, la realidad demográfica del país es diversa, cada entidad presenta características particulares que ocasionan que el envejecimiento poblacional sea vivido de distinta manera. Especialmente en este estudio se analiza la situación de tres estados del país: Nuevo León, Ciudad de México y Jalisco.

La Ciudad de México es la capital del país, también destaca por ser una de las entidades del país que presenta una elevada proporción de personas mayores, dado que del total de su población $14.3 \%$ son personas de 60 años o más; respecto a la esperanza de vida para el 2019 se estima que es de 76.49 años en promedio; por su parte, Jalisco, región ubicada al oeste del país, su población mayor constituye el $10.3 \%$ de la población total en la entidad, con una esperanza de vida media de 75.3 años; finalmente, Nuevo León, que se encuentra al noreste del país, su población adulta mayor representa el $9.8 \%$ de la población total de la entidad, manteniendo una esperanza de vida de 77.06 años (Instituto Nacional de Estadística y Geografía, 2015; Consejo Nacional de Población, 2018).

Estas cifras muestran una fuerte presencia de personas adultas mayores en estas entidades mexicanas, lo cual origina la necesidad de conocer las condiciones de vida de esta población. Este escrito se centra en el tema del apoyo social, y, aunque es un asunto relevante durante todo el curso de vida, en la vejez adquiere nuevos matices pues tiene una estrecha relación con el bienestar de las personas mayores (Arias, 2015). Sin embargo, no todas las personas cuentan con redes de apoyo sólidas, ya que en el contexto mexicano una alta proporción de personas mayores no tienen acceso a pensión, así como tampoco a servicios de salud, por lo que la familia se configura como el principal mecanismo de apoyo en la vejez (Garay y Román, 2016).

\section{POLÍTICA SOCIAL Y VEJEZ EN MÉXICO}

A nivel global el fenómeno del envejecimiento acontece en el marco de una serie cambios y reconfiguraciones, en donde las políticas sociales enfocadas en las personas adultas mayores se estructuran a partir de tensiones, acuerdos, permanencias y transformaciones. Además, son influenciadas por dimensiones no sólo sociales, sino también económicas y políticas; así, estos tres elementos "entretejen y reconfiguran el contexto donde la población envejece y es atendida" (Avalos, 2014: 418).

La intervención del trabajo social no es ajena a la política social, así como a los problemas que enfrentan las personas mayores, de hecho, se encuentran imbricados pues como señala Alayón (2010) lo que se busca es contribuir al me- 
joramiento de las condiciones de vida y el bienestar de la población. Sin embargo, las condiciones actuales distan de ser las mejores, pues en la mayoría de los países de la región existe una ausencia del Estado y poco interés en atender las necesidades de la población.

En México, la construcción e implementación de políticas sociales destinadas a la atención de grupos sociales en situación de vulnerabilidad ha tenido constantes cambios y orientaciones, permeadas en gran medida por los intereses y prioridades de la agenda pública de cada administración de gobierno, la cual cambia conforme se termina las administraciones sexenales; en este sentido, las políticas para la atención de las personas adultas mayores no ha sido una excepción (Vivaldo y Martínez, 2012).

En la actualidad existe el Instituto Nacional de las Personas Adultas Mayores, el cual es el organismo rector de la política nacional a favor de las personas adultas mayores. Sin embargo, para Vivaldo y Martínez (2012), a pesar de las capacidades, atribuciones y obligaciones establecidas en la Ley, este Instituto no ha logrado consolidarse como organismo rector para el diseño de las políticas públicas en México en materia de envejecimiento y vejez.

Una estrategia específica implementada por el Estado en materia de vejez fue el Programa de atención a los adultos mayores de 70 años y más en zonas rurales, en el año 2007, focalizado en población mayor en condiciones de pobreza y de marginación en localidades de hasta 2500 habitantes. Dicho programa se ha ido transformado conforme han cambiado los gobiernos, ha pasado de ser una política focalizada a una política de carácter universal.

En la actual administración de gobierno y bajo los lineamientos del Plan Nacional de Desarrollo 2019-2024, se implementa el Programa para el bienestar de las personas adultas mayores, el cual tiene por objetivo "Contribuir al bienestar social e igualdad de la población adulta mayor, a través del otorgamiento mensual de una pensión no contributiva"; dicho programa está destinado a personas indígenas adultas mayores de 65 años, personas adultas mayores de 68 años y personas mayores de 65 años que hayan sido incorporadas de manera previa; el monto del apoyo económico es de $\$ 1275.00$ pesos mensuales, con entregas bimestrales (Diario Oficial de la Federación, 2019: s/p).

Por lo anterior, es posible señalar que existe la intención de actual gobierno de incorporar los lineamientos establecidos en los acuerdos internacionales, pero habrá que estar atentos al desarrollo, implementación y evaluación de este programa que incorporó el tema de la universalidad, ya que como señala Vivaldo y Martínez (2012:39) en el contexto na- cional "las políticas se han construido a partir de visiones de gobierno y no de Estado".

En definitiva, el envejecimiento poblacional plantea nuevas demandas en relación con la satisfacción de necesidades, el bienestar y la calidad de vida de la población, por lo que, desde la política social y desde el trabajo social la atención de estos aspectos es fundamental para garantizar el apoyo y el respeto de los derechos de las personas mayores y sus familias.

\section{APOYOS FAMILIARES}

La familia es uno de los ámbitos fundamentales para el ser humano, pero su configuración no es estática. Al respecto, Arriagada (2007) enfatiza el surgimiento de familias diversas, cambiantes y desiguales en el marco de grandes cambios estructurales y demográficos, que a su vez también han modificado la organización y la distribución de las responsabilidades dentro y fuera de los hogares.

Con el acelerado proceso de envejecimiento que se vive en el país surgen nuevas configuraciones familiares y, al mismo tiempo, se generan demandas y responsabilidades de apoyo. En México, ante la insuficiencia de los recursos públicos asignados por el Estado para cubrir las necesidades de la población envejecida, las familias han sido quienes han sorteado y solventado las necesidades de la población mayor, ya sea por los vínculos de reciprocidad, por los deberes filiales o morales, por los bienes materiales o por los vínculos emocionales que se han trazado durante el curso de vida.

A diferencia de otras regiones, en México la mayor parte de la población envejecida vive en compañía de sus familiares, no obstante, ello no asegura la satisfacción de las necesidades más apremiantes durante la vejez pues, "cada arreglo residencial no puede garantizarnos un sistema de apoyo y por tanto un nivel de vida aceptable" (Montes de Oca, 2001:132). De esta forma, dada la mayor presencia de miembros envejecidos dentro de los hogares es importante considerar las condiciones en las que las personas están envejeciendo y las demandas que trae consigo el incremento de este grupo poblacional, especialmente en términos sociales, económicos, familiares, de apoyo y de cuidados.

Así, el interés por el tema surge en un contexto transiciones y transformaciones familiares que instan a reflexionar sobre los factores asociados a la recepción de apoyo familiar en la vejez. De acuerdo con algunos autores (Montes de Oca, 2006; Scott y Wenger, 1996), la familia es la base de las diferentes redes de apoyo social. De esta manera, el apoyo familiar juega un papel importante en el bienestar de la po- 
blación, el cual es brindado principalmente por el cónyuge y los hijos, aunque cabe señalar que existen diferencias según el género (Montes de Oca y Macedo, 2013).

En otra investigación realizada por Millán-León (2010) se encontró que hay contrastes de género en el apoyo brindado a las personas mayores, por ejemplo, las transferencias económicas son menores en los hombres en comparación con las mujeres; en el caso de los primeros, éstos generalmente no están acostumbrados a depender económicamente de alguien más. Además, al ser ellos quienes representan mayores proporciones en la población económicamente activa, son visualizados como menos vulnerables económicamente.

Por su parte, Salgado y Wong (2006) señalan con base en una investigación realizada en contextos de pobreza urbana de cuatro ciudades del país (Chilpancingo, Cuernavaca, Culiacán y Guadalajara), que la familia continua fungiendo como la principal proveedora de apoyo, particularmente hacia las personas adultas mayores que presentan condiciones vulnerables, en estos casos las personas se caracterizan por presentar un estado de salud precario, acompañado de una alta prevalencia de enfermedades crónicas, así como una acentuada manifestación de síntomas depresivos.

En este mismo orden de ideas, Garza-Sánchez y González-Tovar (2018) refieren, con base en un estudio realizado en el norte de México, que las mujeres mayores tienen una percepción positiva del apoyo social que reciben, lo que contribuye a su bienestar; además, en el tema del apoyo se encontró que la red familiar es la red de apoyo más cercana, pues las personas mayores se perciben con protección y cariño.

Con el objeto de conocer los apoyos en los hogares con al menos una persona adulta mayor en el Estado de México, Cervantes (2013) analiza las características de las personas que dan y reciben apoyo, los resultados arrojados en el estudio muestran que las mujeres dan y reciben más apoyo en contraste con los hombres; respecto a la edad se observó que entre los 60 y 69 años, tanto hombres como mujeres dan más apoyo del que reciben, sin embargo, conforme se incrementa la edad se tiende a recibir más apoyo del que se brinda; con relación al estado de salud, se determinó que entre las personas que reciben ayuda es más notable el estado de mala salud; en cuanto a la situación laboral se aprecia que hay más hombres empleados que mujeres, en el caso de los primeros de los varones que reciben apoyo $52.8 \%$ no trabaja, en el caso de las mujeres $80.8 \%$.

Respecto al lugar de residencia, según García-Sanz (1998) vivir en comunidades rurales puede generar algunas ventajas, en estos contextos las personas adultas mayores tienen mayores posibilidades de subsanar la soledad, dado que se cuenta con mayor apoyo vecinal que en la ciudad, en esta última se han quebrantado estos lazos, o bien no se realizan con la misma regularidad. En esta misma línea, Rodríguez (2004), señala que residir en un contexto rural, así como envejecer en el mismo, plantea ciertas condiciones favorables tales como un proceso más lento en la disminución de la actividad y una mayor interacción con las redes de apoyo social.

A partir de la revisión de la literatura se muestra que la situación de las redes de apoyo en la vejez es heterogénea, no existe un patrón que determine la existencia o no del apoyo, sin embargo, se evidencia que pueden llegar a presentarse ciertas características que inciden en la manifestación del apoyo. Por lo que en este estudio se plantea conocer ¿cuáles son los factores que intervienen en la recepción de apoyo familiar en las personas adultas mayores residentes en tres entidades del país?

\section{OBJETIVO GENERAL}

Analizar los factores individuales, familiares y contextuales que intervienen en la recepción de apoyo familiar de las personas adultas mayores en tres entidades del país: Nuevo León, Jalisco y Ciudad de México.

\section{METODOLOGÍA}

El presente estudio es de corte cuantitativo, con un diseño transversal, no experimental y ex post facto. La población objetivo está constituida por ciudadanos mexicanos con una edad de 60 años o más, residentes de áreas rurales y urbanas en las entidades federativas de Nuevo León, Jalisco y Ciudad de México.

Se utilizó un muestreo estratificado tomando como referencia el tamaño de la población de 60 años y más en cada uno de los contextos (rural y urbano) de las tres entidades federativas consideradas en el estudio, para ello se consideraron datos de Instituto Nacional de Estadística y Geografía (2010). Las entidades federativas de levantamiento de la información fueron las siguientes: Nuevo León, Jalisco y la Ciudad de México. La muestra es representativa de las personas mayores en cada entidad, misma que quedó conformada por 1152 casos con un nivel de confianza del $95 \%$ y un error de muestreo del $5 \%$.

\begin{tabular}{|lccc|}
\hline \multicolumn{4}{|c|}{ Tabla I. Distribución DE LA Muestra } \\
\hline Entidad & Rural & Urbana & Total \\
\hline Nuevo León & 116 & 268 & 384 \\
\hline Jalisco & 115 & 269 & 384 \\
\hline Ciudad de México & 115 & 269 & 384 \\
\hline
\end{tabular}

Fuente: elaboración propia 
El levantamiento de la información se realizó entre los meses de abril a julio del 2015. Las encuestas se realizaron cara a cara, en lugares de afluencia (plazas públicas, mercados, calles de alto tránsito). El instrumento utilizado fue la Encuesta sobre Condiciones de Vida de las Personas Adultas Mayores en México, 2016, dicha encuesta es la base de un estudio más amplio, pero para fines de este análisis, del instrumento señalado se aborda de forma principal el tema de las redes de apoyo social.

Entre las limitantes del estudio se puede señalar que no es un estudio representativo de las localidades urbanas y rurales, sino de la población adulta mayor en cada entidad. La determinación de la muestra para áreas urbanas y rurales se calculó intencionalmente con tamaños similares (70\% urbano y $30 \%$ rural), de manera que se pudiera comparar.

Para el análisis de la información se utilizó el programa Statistical Packagefor Social Science (SPSS) versión 22.0. En primer lugar, se hizo una exploración de los datos de manera general a través de frecuencia y porcentajes de las caracte- rísticas generales. A partir de los datos encontrados en la primera fase y considerando la literatura revisada se procedió a crear un modelo de regresión logística binaria, con cálculo de odds ratio bruto y ajustado con un intervalo de confianza de $95 \%$ (IC 95\%), siendo mantenidas en el modelo aquellas con $\mathrm{p}<0.01$.

Dados los distintos elementos que inciden en el apoyo hacía las personas adultas mayores en la vejez, tanto individuales, como familiares y contextuales en el siguiente análisis estadístico se integran gran parte de estos factores. Con esto se persigue el objetivo de analizar la intervención de cada uno de estos factores en la recepción de apoyo familiar en la población adulta mayor de tres estados del país, esto mediante una regresión logística binaria, con la cual esta población se divide en dos categorías: población que sí recibe apoyo familiar y población que no recibe, las cuales son distinguidas por la influencia de los factores individuales, familiares y contextuales (variables independientes).

Para los cálculos realizados, dichas variables fueron operacionalizadas de la siguiente manera:

\begin{tabular}{|c|c|c|c|c|}
\hline \multicolumn{5}{|c|}{ TABLA II. OPERACIONALIZACIÓN DE VARIABLES } \\
\hline Variable & & Indicador & Valor final & Tipo de variable \\
\hline $\begin{array}{l}\text { Variable } \\
\text { dependiente }\end{array}$ & Apoyos familiares & Recepción de apoyos familiares & $\begin{array}{l}\text { No recibe }=0 \\
\text { Sí recibe }=1\end{array}$ & Nominal Dicotómica \\
\hline \multirow[t]{4}{*}{$\begin{array}{l}\text { Factores } \\
\text { individuales }\end{array}$} & Sexo & Sexo de entrevistado (a) & $\begin{array}{l}\text { Hombre }=0 \\
\text { Mujer }=1\end{array}$ & Nominal Dicotómica \\
\hline & Edad & Años cumplidos de entrevistado (a) & Años & Numérica \\
\hline & Enfermedades & Tiene enfermedades & $\begin{array}{l}\text { No tiene enfermedades }=0 \\
\text { Sí tiene enfermedades }=1\end{array}$ & Nominal Dicotómica \\
\hline & Limitantes en ABVD & $\begin{array}{l}\text { Tiene limitantes para realizar } \\
\text { actividades básicas de la vida diaria }\end{array}$ & $\begin{array}{l}\text { No tiene limitantes }=0 \\
\text { Sí tiene limitantes }=1\end{array}$ & Nominal Dicotómica \\
\hline $\begin{array}{l}\text { Factores } \\
\text { familiares }\end{array}$ & Hijos & Tiene hijos & $\begin{array}{l}\text { No tiene hijos }=0 \\
\text { Sí tiene hijos }=1\end{array}$ & Nominal Dicotómica \\
\hline \multirow[t]{3}{*}{$\begin{array}{l}\text { Factores } \\
\text { contextuales }\end{array}$} & Situación laboral & Actualmente trabaja & $\begin{array}{l}\text { No trabaja }=1 \\
\text { Sí trabaja =0 }\end{array}$ & Nominal Dicotómica \\
\hline & $\begin{array}{l}\text { Acceso a servicio } \\
\text { médico }\end{array}$ & Tiene derecho a atención médica & $\begin{array}{l}\text { No tiene atención médica }=0 \\
\text { Sí tiene atención médica }=1\end{array}$ & Nominal Dicotómica \\
\hline & Localidad & Lugar de residencia & $\begin{array}{l}\text { Rural }=0 \\
\text { Urbano }=1\end{array}$ & Nominal Dicotómica \\
\hline
\end{tabular}

Fuente: elaboración propia 


\section{RESULTADOS}

Las redes de apoyo familiar en la vejez cobran relevancia, debido a que generalmente son la principal fuente de apoyo ante situaciones complejas, por ejemplo, la aparición de enfermedades, la pobreza, la necesidad de cuidados, entre otros. Así, con base en la revisión de literatura se seleccionaron algunas variables atribuidas a factores individuales, familiares y contextuales con la intención de analizar la influencia en éstos en la recepción de apoyo familiar de las personas adultas mayores residentes en tres entidades de México. Para ello se ajustó un modelo de regresión logístico binario.

En primer lugar, se analizaron los datos a partir de la prueba de ji-cuadrada, esto para ver la asociación entre la variable dependiente y las variables independientes. De acuerdo con los resultados de la prueba, al hacer un análisis bivariado, se puede afirmar con un $\mathbf{9 5 . 0 \%}$ de confianza que la recepción de apoyos familiares en las personas adultas mayores se encuentra asociada de manera estadísticamente significativa (Sig. < 0.01) con las siguientes variables: la localidad de residencia, el tener hijos y la situación laboral. En tanto, en variables como el sexo, las limitantes en ABVD, el diagnóstico de enfermedad, el tener ingresos por pensión o jubilación y tener vivienda propia, no resultaron estar asociadas de manera estadísticamente significativa.

$\begin{aligned} & \text { TABLA III. AsOCIACIÓN ENTRE LA RECEPCIÓN DE APOYO FAMILIAR } \\
& \text { DE PERSONAS ADULTAS MAYORES Y FACTORES INDIVIDUALES, } \\
& \text { FAMILIARES Y CONTEXTUALES }\end{aligned}$
\begin{tabular}{lcrc} 
Variables independientes & Valor & gl & Sig. \\
\hline Sexo & 3.409 & 1 & 0.065 \\
\hline Enfermedades & 0.122 & 1 & 0.727 \\
\hline Limitantes en ABVD & 3.777 & 1 & 0.052 \\
\hline Localidad de residencia & 45.020 & 1 & 0.000 \\
\hline Tiene hijos & 103.122 & 1 & 0.000 \\
\hline Recibe ingresos por jubilación & 2.172 & 1 & 0.141 \\
\hline Tiene vivienda propia & 0.002 & 1 & 0.965 \\
\hline Actualmente trabaja & 34.297 & 1 & 0.000 \\
\hline Estadísticos globales & 175.764 & 8 & 0.000 \\
\hline
\end{tabular}

Fuente: elaboración propia con base en la Encuesta sobre Condiciones de Vida de las Personas Adultas Mayores en México, 2016.

Ahora bien, después de conocer la asociación entre las variables se procedió a elaborar el modelo de regresión logística binaria, en donde además de incluir las variables que resultaron estar asociadas en la prueba ji-cuadrada se integró la variable edad, así mismo se incorporaron las variables sexo y limitantes en $A B V D$, por ser dos de las variables que en la literatura se han reportado estar asociadas con la transferencia de apoyos.

\begin{tabular}{|lc|}
\hline \multicolumn{2}{|c|}{ TABLA IV. RESULTADOS DE LA REGRESIÓN LOGÍSTICA BINOMIAL } \\
PARA MODELAR LA PROBABILIDAD DE RECIBIR APOYOS EN LA VEJEZ \\
\hline Factores & Exp. (B) \\
\hline Sexo & $1.451^{*}$ \\
Mujer & 1.000 \\
\hline Hombre & $1.048^{*}$ \\
\hline Edad & 0.995 \\
\hline Enfermedades & 1.000 \\
Sí tiene enfermedades & 0.903 \\
No tiene enfermedades & 1.000 \\
\hline Limitantes en ABVD & \\
Sí tiene limitantes & $6.910^{*}$ \\
No tiene limitantes & 1.000 \\
\hline Tiene hijos & \\
Sí tiene hijos & $3.073^{*}$ \\
No tiene hijos & 1.000 \\
\hline Localidad de residencia & $1.934^{*}$ \\
Rural & 1.000 \\
Urbana & \\
\hline Situación laboral & \\
No tiene trabajo & \\
\hline
\end{tabular}

${ }^{*} \mathrm{p}<0.01$

Fuente: elaboración propia con base en la Encuesta sobre Condiciones de Vida de las Personas Adultas Mayores en México, 2016.

Al analizar los factores individuales, respecto a la variable sexo se encontró que el ser mujer adulta mayor incrementa la probabilidad de recibir apoyos en $45.0 \%$ en contraste con los hombres. Esto coincide con lo expresado por Scott y Wenger (1996), quienes señalan que la diferencias de género inciden en la constitución de las redes de apoyo en la vejez; por ejemplo, en el caso de las mujeres como gran parte de su vida la dedicaron a la crianza y a la realización de actividades dentro del hogar cuentan con una mayor posibilidad de desarrollar relaciones sociales que suelen prolongarse hasta la vejez; por lo que respecta a los hombres, dado que éstos regularmente centraron su vida social en cuestiones relacionadas con el trabajo, presentan mayor dificultad para integrarse al vecindario y a la familia cuando se han jubilado o bien, cuando ya no le es posible desempeñar otras actividades.

Con relación a la edad, se observa que a mayor edad se incrementa en $4.8 \%$ la probabilidad de recibir apoyos. Autoras como Montes de Oca (2003) ya han señalado que parece haber una tendencia que apunta que, mientras mayor es la edad de las personas mayores, se incrementa la probabilidad 
de que aparezcan enfermedades y dependencia, así como una menor participación en actividades grupales.

Cabe destacar que se decidió integrar en el modelo las variables enfermedades y limitantes en $A B V D$, ya que son dimensiones de interés para fines de este estudio, debido a que en la literatura están reportadas como factores que inciden en la recepción de apoyos, sin embargo, éstas no resultaron ser estadísticamente significativas en el modelo de regresión logística binaria, así como en la prueba ji-cuadrada. Lo cual indica que en esta población el tener enfermedades y limitantes en ABVD no son factores que expliquen la recepción de apoyos en las personas adultas mayores. Esto pudiera estar relacionado con el hecho de que la población envejecida que recibe el apoyo no reconozca la ayuda que le brindan sus familiares particularmente ante estas situaciones y lo vea como un deber moral de la familia.

En cuanto a los factores familiares, únicamente se incluyó la variable tiene hijos, misma que de acuerdo con el modelo de regresión logística binomial presenta un valor de 6.910 odds ratio, lo cual significa que las personas que sí tienen hijos tienen una probabilidad casi 7 veces mayor de recibir apoyo familiar en comparación con las personas que no tienen hijos. Este dato es consistente con lo referido en la literatura, pues según algunos autores (Montes de Oca, 2006; Scott y Wenger, 1996; Universidad de Costa Rica-CONAPAM, 2008), en la vejez la familia es la base de las diferentes redes de apoyo social, no importa si es corresidente o no, en este tipo de red los principales proveedores de apoyo son los hijos y en algunos casos en cónyuge, de ahí la importancia de esta variable.

Dentro del ámbito familiar el apoyo de los hijos es relevante para el bienestar de la población mayor, no obstante, también hay que poner en contexto que se ha reducido la fecundidad y la natalidad, lo que ha influido en que las familias tiendan a ser más pequeñas, esto incide también en el tamaño de la red familiar. Por otra parte, algunos estudios ya han señalado que el hecho de tener hijos no garantiza la presencia de apoyo, por lo que es importante formar parte de otras redes, por ejemplo, las redes de amigos, de vecinos o las comunitarias (Montes de Oca, 2003).

Por lo que concierne a los factores contextuales, fueron consideradas dos variables, en primer lugar, la variable localidad de residencia, en ésta se muestra que la odds ratio tiene un valor de 3.073, lo cual revela que las personas adultas mayores que residen en localidades rurales tienen 3 veces más probabilidad de recibir apoyo por parte de su familia en contraste con las personas que habitan en lugares urbanos. Esto parece indicar que en las comunidades rurales continúan prevaleciendo lazos de solidaridad hacia los miembros envejecidos, en tanto en los lugares urbanizados la posibilidad de recibir apoyo es menor, lo cual puede estar relacionado con la exigencia de las dinámicas laborales.

Finalmente, al considerar la variable situación laboral se observa que la odds ratio presenta un valor de 1.934. Esto confirma que las personas adultas mayores que no se encuentran trabajando tienen casi 2 veces más probabilidad de recibir apoyo en comparación con las que sí se encuentran trabajando. En el estudio se encontró una diferencia importante entre quienes trabajan y quienes no lo hacen $(36.7 \%$ y $63.3 \%$ respectivamente); además, los resultados revelan que entre las personas que reportaron que sí trabajan, la mayoría son hombres.

El tema de la participación en el mercado laboral es fundamental en todo el curso de vida, pero en la vejez adquiere ciertos matices, pues en algunos casos lo hacen por necesidad ante carencias económicas, también como una manera de mantenerse activos o para contribuir en el gasto familias, pues no sólo son receptores también en algunos casos llegan a proveer.

\section{CONSIDERACIONES FINALES}

El contexto en el que las personas adultas mayores están envejeciendo es complejo, dadas las diversas transformaciones ocurridas en términos demográficos y familiares. Al estudiar en este artículo el apoyo familiar se buscó determinar cuáles son los factores inciden en la recepción de apoyo familiar. Con el análisis realizado a través de la regresión logística binaria se logró constatar que factores como el sexo, la edad, el tener hijos, la localidad de residencia y la situación laboral tienen una influencia importante en la manifestación del apoyo familiar. Cabe señalar que, de los factores individuales, el que más incide es el sexo, puesto que se encontró que las mujeres tienen mayor probabilidad de recibir apoyo en comparación con los hombres.

Las diferencias de género en la recepción del apoyo familiar pueden estar vinculados con los roles y las representaciones sociales que se han entretejido en torno al género y que se encuentran fuertemente vinculadas con la división sexual del trabajo (mujeres asociadas con la esfera reproductiva, mientras que los varones con la esfera productiva), así como las relaciones y vínculos que se han construido a lo largo del curso de vida. Por su parte, en los factores familiares, aunque sólo se incluyó el tener hijos, esta variable resultó tener una fuerte influencia en la recepción de apoyos familiares, el resultado obtenido es consiste con lo expresado en otros 
estudios, pues dentro del apoyo familiar está reportado que los hijos son los principales proveedores de apoyo.

Esto no significa que todas aquellas personas mayores que tienen hijos cuentan con apoyo, pero sí existe una mayor probabilidad de ser susceptible de apoyo. En este aspecto hay que profundizar más, puesto que las personas mayores no sólo son receptores de apoyo, sino que establecen vínculos de reciprocidad con los hijos, a través del cuidado de los nietos, provisión de comida, vivienda, apoyo económico o emocional, entre otros.

De los factores contextuales, la situación laboral y la zona de residencia son variables que influyen en la recepción de apoyos familiares en la vejez, esto resulta ser un hallazgo importante, pues confirma que ante situaciones de vulnerabilidad económica (no tener ingresos por trabajo), la familia es un soporte para las personas envejecidas. La escasez de condiciones laborales dignas y favorables propician que la vejez sea concebida como una etapa de inseguridad, no sólo económica sino también social. En ese sentido, la actuación del Estado ha sido limitada, por lo que principalmente la familia es la red que apoya cuando se enfrenta la falta de recursos económicos, prestaciones laborales y seguridad social.

Con respecto a la zona de residencia, se evidencia que en las zonas rurales existe una mayor probabilidad de ser sujeto de apoyo familiar en la vejez, lo que significa que prevalecen lazos de solidaridad. No obstante, teniendo como referencia el contexto actual con tendencias capitalistas y neoliberales el apoyo familiar puede llegar a sufrir afectaciones debido a la migración masiva del campo a la ciudad, la inseguridad en el retorno de los migrantes, las condiciones de pobreza que prevalecen en gran parte de las regiones rurales del país y, especialmente debido a la fuerza de los imperativos económicos asociados con la productividad, la competitividad y el individualismo (Flores, 2019).

En síntesis, el estudio muestra un panorama general en torno a los principales factores asociados a la recepción del apoyo familiar en la vejez, esto en distintas entidades del contexto mexicano. Pero creemos que hay que continuar profundizando en cada uno de los factores referidos y contextualizarlos geográfica, generacional y socialmente. Consideramos que el acercamiento teórico y metodológico desde la perspectiva del curso de vida puede contribuir a comprender la construcción de las redes de apoyo desde edades tempranas. Generalmente los estudios los hemos abocado a la etapa de la vejez, pero recordemos que ésta se construye desde etapas previas.

Finalmente, otro aspecto que es importante enfatizar es que, aunque en este análisis nos enfocamos en los factores de la recepción de apoyo familiar, las personas mayores no sólo son receptores también brindan y proveen distintos tipos de apoyo, que repercute en el bienestar de la familia. Y, aunque la familia es la principal fuente de apoyo en la vejez, no se puede obviar que también existe una corresponsabilidad del Estado que no se está cumpliendo completamente, pues continúan existiendo condiciones que vulneran la vida de las personas mayores.

\section{REFERENCIAS BIBLIOGRÁFICAS}

Alayón, N. (2010). Acerca de la caracterización de la Política Social y la práctica del Trabajo Social. Revista Trabajo Social UNAM, 1, 8-21.

Avalos, R. (2014). Globalización y políticas sociales hacia la vejez: algunas consideraciones para el caso de la Ciudad de México. En, V. Montes de Oca (Coord.), Vejez, salud y sociedad en México Aproximaciones disciplinarias desde perspectivas cuantitativas y cualitativas (pp. 417-440). México: UNAM, Instituto de Investigaciones Sociales, Secretaría de Desarrollo Institucional, Seminario Universitario Interdisciplinario sobre Envejecimiento y Vejez.

Cervantes, L. (2013). Apoyos en los hogares con al menos un adulto mayor en el Estado de México. Papeles de Población, 19, 63-92.

Consejo Nacional de Población. (2015). Datos de proyecciones. Recuperado de http://www.conapo.gob.mx/es/CONAPO/Proyecciones_Datos

Consejo Nacional de Población. (2018). Protocolo San Salvador, Esperanza de vida (ambos sexos). Recuperado de https://datos.gob.mx/busca/dataset/proyecciones-de-la-poblacion-de-mexico-y-de-las-entidades-federativas-2016-2050/resource/d0c5fef2-642f-41f0-9e22-b8cbd67bf4b3.

Diario Oficial de la Federación. (2019). Acuerdo por el que se emiten las Reglas de Operación de la pensión para el bienestar de las personas adultas mayores, para el ejercicio fiscal 2019. Recuperado de https://dof.gob.mx/nota_ detalle. php?codigo $=5551445 \&$ fecha $=28 / 02 / 2019$.

Flores, R.M. (2019). Modernización y redes de apoyo familiar en la vejez rural. En S. Garay (Coord.) Formas de envejecer: condiciones y necesidades de las personas mayores (pp. 101-121). México: Universidad Autónoma de Nuevo León.

Garza-Sánchez, R.I. y González-Tovar, J. (2018). El apoyo social en la vejez: diferencias por sexo en una muestra del norte de México. Interacciones: Revista de Avances en Psicología, 4, 191-198. 
Garay, S. y Román, A. (2016). Condiciones de vida de las personas mayores beneficiarias del programa de apoyo al adulto mayor en Nuevo León. Iberoforum. Revista de Ciencias Sociales de la Universidad Iberoamericana, 22, 98-116.

García-Sanz, B. (1998). Los mayores y el mundo rural. Recuperado de http://www.caritas.es/imagesrepository/CapitulosPublicaciones/590/07\%20-\%20LOS\%20MAYORES\%20EN\%20EL\%20MUNDO\%20RURAL.PDF.

González, K. D. (2015). Envejecimiento demográfico en México: análisis comparativo entre las entidades federativas. México: CONAPO. Recuperado de http://www.conapo.gob.mx/ work/models/CONAPO/Situacion_Demografica_De_Mexico/2015/HTML/files/assets/basic-html/page-4.html

Help Age (2015). Índice global de envejecimiento. Age watch 2015. Recuperado de https://www.helpage.org/la/publicaciones/publicaciones2/publicaciones-destacadas/.

Instituto Nacional de Estadística y Geografía. (2015). Encuesta Intercensal 2015. Recuperado de http://www.inegi. org.mx/est/contenidos/Proyectos/encuestas/hogares/especiales/ei2015/.

Montes de Oca, V. (2001). El envejecimiento en México: un análisis sociodemográfico de los apoyos sociales y el bienestar de los adultos mayores. México D.F: El Colegio de México.

Montes de Oca, V. (2003). Redes comunitarias, género y envejecimiento. Notas de población año XXIX, n 77, Santiago de chile: Naciones Unidas - CEPAL. Recuperado de https://repositorio.cepal.org/bitstream/handle/11362/12753/np77139174_es.pdf?sequence=1\&isAllowed=y . 139-174
Montes de Oca, V. (2006). Redes comunitarias, género y envejecimiento. México: Colección Cuadernos de Investigación, Instituto de Investigaciones Sociales-UNAM.

Montes de Oca, V. y Macedo, L. (2013). Redes sociales como determinantes de la salud. Recuperado de http://www.inger.gob.mx/bibliotecageriatria/acervo/pdf/envejecimiento_y_salud_11.pdf

Millán-León, B. (2010). Factores asociados a la participación laboral de los adultos mayores mexiquenses. Papeles de población, 16, 93-121.

Rodríguez, P. (2004). Envejecimiento en el mundo rural: Necesidades singulares, políticas específicas. Boletín sobre el envejecimiento. Perfiles y tendencias, 11, 1-26. Recuperado de http://www.imserso.es/InterPresent1/groups/ imserso/documents/binario/boletinopm11.pdf.

Salgado, N. y Wong, R. (2006). Envejeciendo en la pobreza urbana: conclusiones e implicaciones para la salud de los ancianos. En: N. Salgado y R. Wong, Envejecimiento, pobreza y salud en población urbana. Un estudio en cuatro ciudades de México (pp. 155-161). Morelos, México: Instituto Nacional de Salud Pública.

Scott, A. y Wenger, G.C. (1996). Género y redes de apoyo social en la vejez. En S. Arber y J. Ginn, Relacion entre Género y Envejecimiento (pp. 221-240). Madrid: Narcea.

Vivaldo, M. y Martínez, A. (2012). La política pública para el envejecimiento en México. Historia, análisis y perspectivas. En: L.M. Gutiérrez-Robledo y D. Kershenobich- Stalnikowitz (Coord.), Envejecimiento y salud: una propuesta para un plan de acción (pp. 27-41). México: Universidad Nacional Autónoma de México. 